\title{
109. Electron Microscopic Studies of Ependymal Cell and Choroid Plexus in Experimental Head Injury
}

\author{
Hironobu Shimada, Yousuke AraI, Mamoru TaKagaki \\ and Seiji HosodA \\ Department of Surgery, Juntendo University School of Medicine
}

\begin{abstract}
Mongrel dogs were used as experimental animals. Closed head injury was made on the animals, by means of the negative $G$ acceleration, which were placed in the centrifugal apparatus. The animals were sacrificed between 30 minutes and 3 months after the iujury. Specimens were removed from the wall of lateral ventricle and chroid plexus of lateral or 4th ventricle, and then fixed by $2 \%$ osmium-tetroxid in bufferd phosphatic acid solution for electron microscopic study.

During 24 to 48 hours after the head injury, microvilli of the ependymal cell increased and swelled markedly, smooth surfaced endoplasmic reticulum and Golgi apparatus developed, and dilated. Vesicle was numerous near the plasma membrane of free surface in the epithelium.

On the other hand, mitochondria of chroid plexus epithelium increased in density within 6 hours after the injury. After 24 or 48 hours, mitochond ria swelled, rough surfaced endoplasmic reticulum dilated.

In adition, occasionally poly poid process enla rged, microapocline secretion and pinocytosis developed.

After 7 days or more, the findings enumerated above were not remarkable.
\end{abstract}

\section{Attempts to Scan Brain Edema Utilizing $\mathbf{H g}^{203}$-Neohydrin in Patients with Head Injury}

\author{
Hajime Nagal, Kazuhiro Furuse, Kazuhiko OKamura, \\ Akira Shintani, Yoshihiko OKa, Tatsuya Kobayashi \\ and Atsushi IKEYama \\ 2nd Dept. of Surgery, School of Medicine, Nagoya University
}

Brain scanning for brain tumor utilizing $\mathrm{Hg}^{203}-\mathrm{Neohydrin}$ has been employed in the U.S.A. since Bender and Blau introduced this material. Many reports have confirmed the advantages of this material as compared with the other radioactive isotopes, that is, scans can be made with this 